\title{
Chronic Kidney Disease in India: Challenges and Solutions
}

\author{
S.K. Agarwal ${ }^{a}$ R.K. Srivastava ${ }^{b}$

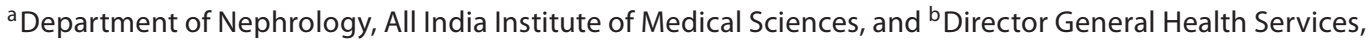 \\ Ministry of Health and Family Welfare, Government of India, New Delhi, India
}

\section{Key Words}

Chronic kidney disease, India $\cdot$ Chronic kidney disease, prevention $\cdot$ Renal transplant $\cdot$ CAPD $\cdot$ Hemodialysis

\begin{abstract}
Chronic diseases have become a major cause of global morbidity and mortality even in developing countries. The burden of chronic kidney disease (CKD) in India cannot be assessed accurately. The approximate prevalence of CKD is 800 per million population ( $\mathrm{pmp}$ ), and the incidence of end-stage renal disease (ESRD) is $150-200$ pmp. The most common cause of CKD in population-based studies is diabetic nephropathy. India currently has $820+$ nephrologists, $710+$ hemodialysis units with 2,500+ dialysis stations and 4,800+ patients on CAPD. There are 172+ transplant centers, two-thirds of which are in South India and mostly privately run. Nearly 3,500 transplants are done annually, the total number of cadaver donors being approximately 700 till now. Thus, taken together, nearly $18,000-20,000$ patients (10\% of new ESRD cases) in India get renal replacement therapy. The cost of single hemodialysis varies between USD 15 and 40 with an additional cost of erythropoietin being USD 150-200/month. The cost of CAPD using a ' $Y$ ' set with 3 exchanges/week is USD 400/month. The cost of the transplant procedure in a state-run hospital is USD 800-1,000, and the cost of immunosuppression using tacrolimus, steroid and mycophenolate is USD 350-400/month. Until recently, the government did not recognize CKD/ESRD as a significant problem in India. However, some illustrious activities in relation to CKD brought at-
\end{abstract}

tention of the media and policymakers to this very common but till now deprived group of diseases. On the one side the government has initiated a process by which it is planning to establish stand-alone hemodialysis units in the country to increase the facilities at an affordable cost, and on the transplant side it had launched a National Organ Transplant Program to facilitate transplantation on a national scale. Hemodialysis program is halfway to being implemented. Thus, in India there is still a long way to go with respect to CKD. Until then, in a country like India, screening of high-risk individuals for CKD and the risk factors is the best bet.

Copyright $\odot 2009$ S. Karger AG, Basel

Chronic diseases have become a major cause of global morbidity and mortality. Earlier considered to be a health problem only in developed countries, 4 out of 5 chronic disease deaths now occur in low- and middle-income countries [1]. In India the projected number of deaths due to chronic diseases will rise from 3.78 million in 1990 ( $40.4 \%$ of all deaths) to an expected 7.63 million in 2020 (66.7\% of all deaths) [1]. Traditionally, health programs for prevention of chronic diseases have mainly focused on hypertension, diabetes mellitus and cardiovascular disease (CVD), however, the increase in the prevalence of chronic kidney disease (CKD) progressing to end-stage renal disease (ESRD) and the consequent financial burden of renal replacement therapy (RRT) $[2,3]$ in both developed as well as developing nations has highlighted the importance of CKD and its risk factors. The CKD

\section{KARGER}

Fax +4161306 1234 E-Mail karger@karger.ch www.karger.com
(C) 2009 S. Karger AG, Basel $1660-2110 / 09 / 1113-0197 \$ 26.00 / 0$

Accessible online at:

www.karger.com/nec
Dr. S.K. Agarwal

Professor of Nephrology, All India Institute of Medical Sciences

New Delhi 110029 (India)

Tel. +919811 434 836, Fax +91 1126588663

E-Mailskagarwal58@yahoo.co.in 
burden is increasing rapidly worldwide. At the end of $2004,1,783,000$ patients worldwide were receiving treatment for ESRD, of which $77 \%$ were on dialysis and $23 \%$ had a functioning renal transplant (RT), and this number is increasing at a rate of 7\% every year. If the current situation prevails, the global ESRD population will exceed 2 million by the year 2010 [2]. The average incidence of ESRD in developing countries is 150 per million population (pmp), which is lower than what is reported in the developed world. This has been attributed to racial and ethnic diversity, which is also reflected in the disparity in the incidence of ESRD between different populations within the developed nations [4].

\section{Chronic Kidney Disease in India}

In the absence of a renal registry, the exact disease burden of CKD/ESRD in the Indian population cannot be assessed accurately. In the most representative population-based study from North India, using a multistage cluster sampling technique in which serum creatinine and urine samples were examined in every subject studied, the prevalence of CKD stage 3 and beyond was found in $0.79 \%$ subjects out of 4,972 examined [5]. In this study, CKD diagnosis was based on a repeat sample after 2-3 months to be sure of chronicity of renal disease. However, the diagnosis of renal failure was based on serum creatinine alone $(>1.8 \mathrm{mg} / \mathrm{dl}$, upper limit of normal for the laboratory), which is likely to underestimate the magnitude of CKD. This study also evaluated the prevalence of risk factors for CKD, like diabetes, hypertension, renal stone disease, etc. While extrapolating, the authors concluded that the prevalence of ESRD in India will be $785 \mathrm{pmp}$ and the incidence of ESRD will be $160 \mathrm{pmp}$. The commonest cause of CKD in this population-based study was diabetic nephropathy responsible for $41 \%$ cases. Another study from South India reported a prevalence of impaired kidney function (defined as eGFR $<80 \mathrm{ml} / \mathrm{min}$ calculated on the basis of abbreviated MDRD) to be 8.6 per thousand after screening a population of 25,000 and then 13.9 per thousand population when they subsequently screened another 21,500 people in an adjacent area in a new survey. The prevalence of any type of renal disease (not CKD) was seen in $0.68 \%$ and CKD was seen in $0.16 \%$ in the initial survey $[6,7]$. This study also evaluated diabetes and hypertension, the two major risk factors for CKD. However, there was a difference when compared to the study from North India [5] in so far as serum creatinine was not done in every subject. Further, there was no mention of whether creatinine was repeated after the first screening to be sure of CKD. Another recent study in an urban population from the Central India revealed a similar disease burden as in the extrapolated disease burden from a North Indian study [5] with average crude and age-adjusted incidence rates of ESRD of 151 and $232 \mathrm{pmp}$, respectively [8]. However, this study was limited by the possibility of referral bias and population migration, since it was based on ESRD patients evaluated in a particular hospital and with a premise that all ESRD patients in that population area were coming to this particular hospital. This study had no mention of risk factors for CKD, though the patients were evaluated in the hospital. Further, the primary aim of this study was to assess ESRD and not all CKD patients. The domiciliary screening program for CKD by a trust in South India has reported the prevalence of CKD stage 5 to be 0.87 per thousand ( 870 per million) [9], which is also very similar to extrapolation of an earlier study from North India [5]. In addition to these three community-based studies, there are few hospital-based studies on CKD/ESRD which are restricted to primarily commenting on the etiology of CKD patients presenting to the hospital [10-13]. There are some differences among these studies which are difficult to resolve, as differentiating chronic glomerulonephritis from chronic interstitial disease is very difficult once a patient comes at a stage where a definitive diagnosis cannot be made. However, one thing was certain that diabetes as a cause of CKD in these hospital-based studies was nearly $30 \%$ of all cases. In another hospital-based study, in which data was collected from 48 hospitals representing the whole of India, the prevalence of CKD stage 3 and beyond was found to be approximately $0.8 \%$ [14].

Another source of information on CKD, particularly the pattern of CKD patients presenting to the hospital setting, is a pilot project that was initially started by a group of nephrologists, later the project received endorsement from the National Society of Nephrology. The project, called the 'Indian CKD Registry', initiated in June 2005, aimed at finding out many issues related to CKD in India, including the spectrum of diseases causing CKD. It started with 10 centers as a pilot project involving only adult nephrologists, but currently 152 centers are contributing data to this registry, and recently pediatric nephrologists also have agreed to join. Up until now, the data of approximately 30,000 subjects is in database. Males constitute nearly $70 \%$, with the mean age being approximately 45-50 years of the adult population. The majority of patients were in stage CKD 4-5 groups (70\%). Diabetes mellitus as the cause of CKD was seen most commonly 
in approximately $30 \%$ of the patients, type 2 being responsible in $97 \%$ of the cases and duration of diabetes being $<10$ years in $40 \%$ of the cases. CVD was seen more commonly as the stage of CKD progressed; $0.7 \%$ in stage 1 to $43 \%$ in stage 5 . This registry, as it is now, has some limitations, where point data is captured when the patient comes to hospital for the first time. As of now, there is no follow-up data. The data is also affected as the physician or some nephrologists already treat patients before the registry captures patient's data. This is likely to affect some of the variables. All the data of this registry has until now not been published, however, a website does exist (www.ckdri.org).

Further data generated on CKD in India comes from a study entitled 'Screening and Early Valuation of Kidney Disease' (SEEK), which was started in 2006 by a group of nephrologists, primarily initiated by nephrologists from Brigham \& Women's Hospital \& Harvard Medical School, USA, and several other Indian nephrologists. In addition to determining the prevalence of CKD in India, this study also has very enthusiastic aims and objectives like determining the prevalence of causes and complications of $\mathrm{CKD}$, characterizing the risk factors for $\mathrm{CKD}$, developing predictive risk factor models for CKD and the complications relevant to the Indian population, developing educational material in local languages to educate the population about kidney disease and changing the undergraduate curriculum in relation to CKD. This is primarily a camp approach for inclusion of subjects, although the occasional center claims to have adopted a domiciliary approach. The SEEK data has been presented at the Annual Conference of the Indian Society of Nephrology for the last 2 years. At the last presentation, approximately 6,000 adult subjects from 21 centers from 53 community camps had been screened. Serum creatinine and urine examination could be obtained in $93 \%$ of the patients. This study reported a very high prevalence of $17.4 \%$ of CKD using the standardized eGFR formula, 17-fold higher than other community-based reports from India. In the presentation, there was no mention of criteria to define CKD like repeating a sample of creatinine to be sure of chronicity of kidney disease. This study also evaluated some of the risk factors of CKD like diabetes and hypertension. None of the SEEK data is till now published. It is important to mention here that in the majority of studies mentioned above, eGFR has been calculated using the abbreviated MDRD formula, which has not been validated in the Indian population. However, as of now, it is the only possible method of assessing GFR in community-based studies in India.

CKD in India

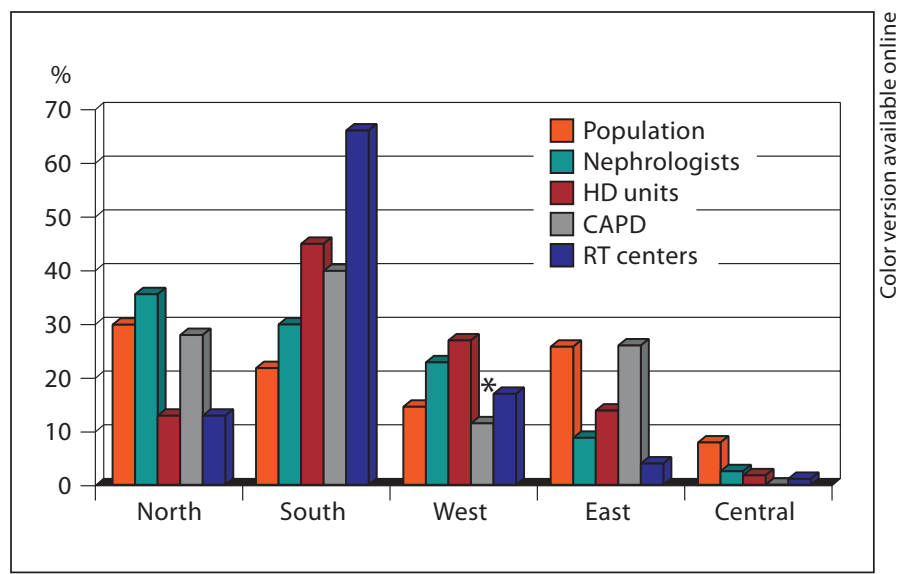

Fig. 1. RRT in India by region. * CAPD in West India also includes Central India data.

Combining all the available literature, both published and unpublished, from various sources, it will not be unwise to comment that the yearly incidence of ESRD in India is approximately $150-200 \mathrm{pmp}$ and diabetes is also an important cause of CKD in approximately $30-40 \%$ of the patients. Further, with current life expectancy being 63 years and increasing more so in the future, with time the magnitude of CKD is going to increase even further. Community studies, like screening for CKD, involve huge costs. The Indian Council of Medical Research funded a 3 -year study in North India, resulting in costs of USD 30,000 , or USD 6 per subject. Any further study to determine the magnitude of the problem of CKD/ESRD in India must therefore be multicentric, involve all regions of India, both rural and urban populations, and follow robust community-based epidemiological strategies, otherwise we are likely to get data which will be no different to what is already known from the above literature.

\section{Challenges in CKD Management in India}

\section{RRT Facilities}

For the treatment of any disease, availability of therapy and its affordability are two important issues. Before discussing the cost of RRT in India, we should review the availability of Indian RRT facilities. India currently has $820+$ nephrologists; of these, 35.5, 30, 23, 9 and 2.5\% are distributed in North, South, West, East and Central India, respectively (fig. 1). Overall, there are $710+$ hemodialysis units. The distribution of units according to state is shown in figure 1. Within these hemodialysis units there are 
Table 1. Requirement (175 pmp), facilities and costs of RRT in India

\begin{tabular}{llcll}
\hline $\begin{array}{l}\text { Facili- } \\
\text { ties }\end{array}$ & Method & $\begin{array}{l}\text { Per million } \\
\text { population }\end{array}$ & $\begin{array}{l}\text { Public } \\
\text { hospital }^{1}\end{array}$ & $\begin{array}{l}\text { Private } \\
\text { hospital }^{1}\end{array}$ \\
\hline 1 & Hemodialysis $^{2}$ & 8.5 & 9,000 & 14,000 \\
2 & CAPD $^{3}$ & 1.7 & 10,000 & 14,000 \\
3 & Renal transplantation $^{4}$ & 2 & 5,600 & 12,000 \\
4 & All RRTs & 12.5 & & \\
\hline
\end{tabular}

${ }^{1}$ Average costs of therapy in USD/year are indicated for either public or private hospitals.

${ }^{2}$ Hemodialysis cost is based on twice a week + erythropoietin 8,000 units/week + consultation charges in a private hospital.

${ }^{3}$ CAPD cost is based on 3 exchanges with a ' $Y$ ' set + erythropoietin 8,000 units/week + consultation charges in a private hospital.

${ }^{4}$ Transplant includes surgery cost + tacrolimus with mycophenolate regimen without induction and cytomegalovirus prophylaxis.

$2,500+$ dialysis stations, with an average number of stations per unit being 3 (range 2-24). With an average number of dialyses per station being twice a day and the average number of dialyses being twice a week, we can reasonably calculate that nearly 15,000 (7-8\% of ESRD incidence) patients are being dialyzed at any one time by hemodialysis modality. Nearly $85 \%$ of these centers are privately run and perform both transplant-oriented dialysis as well as maintenance hemodialysis (MHD). As opposed to the private sector, the government sector cannot afford to provide MHD, and thus only operates RT-oriented hemodialysis facilities. The very first CAPD in India was initiated in 1990, and currently approximately 5,000 patients are on such therapy. Although CAPD is becoming popular and is being used more frequently, it still has a long way to go. In a large number of patients, CAPD is not considered a choice of therapy, but is the last resort. This can be borne by the fact that although $3,200+$ new catheter insertions were done in 2007 , by the end of June 2008, only 4,800+ patients were still on such therapy. This suggests that a large number of patients actually drop out of CAPD therapy due to a combination of reasons like death while on therapy, technique failure, stopping therapy due to economic reasons and getting a RT. The data on percentage of each reason is not very confirmatory, but an approximate guess is that the most common cause is death while on therapy. RT is the best modality of treatment for ESRD patients. It is not only cheaper in the long run, but also provides better survival and a good quality of life. In the RT scene, India has $172+\mathrm{RT}$ centers, mostly on a private basis, and nearly 3,500 RTs are done annually. The distribution of RT centers by geographical region is shown in figure 1 . In the absence of a well-organized cadaver program, living donors constitute the major donor source in India, and unfortunately a large number of them are unrelated. Unrelated RT is more common in private institutions as compared to government-run hospitals. Being against the law in a large number of cases, the results and details of these unrelated transplants are not published in peer-reviewed journals. In spite of an organ transplant bill being passed in 1994, until now only approximately 700 cadavers RTs have been performed in India. In the absence of organized cadavers RTs and the lack of family donors in a large number of situations, it may be difficult to totally stop all unrelated RT on the pretext that it is unethical. Taken together (3,500 RTs + 15,000 MHD + 3,000 CAPD in a year), nearly 21,500 patients (approximately $10 \%$ of new ESRD cases) get RRT. Thus, it is clear that considering the magnitude of the problem of CKD/ESRD, the availability of RRT is limited and also not distributed equally in different parts of the nation (table 1).

The data on the number of physicians getting postdoctorate training in nephrology in India is disheartening. There are $42+$ doctorates in medicine in nephrology seats where physicians get entry by an all-India basis entrance examination. Similarly, the National Board of Examinations takes $46+$ physicians for training for the Diplomate National Board in nephrology at various institutions, mainly private. Both courses last 3 years, after which the trained physician is qualified as a nephrologist to provide RRT.

\section{Cost of Renal Replacement Therapy}

As anywhere in the world, RRT costs are exuberantly high in India, too. With India's per capita gross national product being USD 724 and the average expense of various states and central government for health costs being approximately USD 10.0, the government expects that the cost of RRT should be borne by the individual himself. However, there is some financial support by the government to a small number of patients, and the cost of therapy in a government-run set-up is significantly subsidized. The cost of MHD for a single session varies from USD 15 to 40 between government-run and private hospitals. This excludes the cost of erythropoietin, which is approximately USD $150-200 /$ month, as the majority gets between 4,000 and 8,000 units of erythropoietin every week [15]. 
The cost of CAPD using a 'Y' set with 3 exchanges/week, which most of patients in India do, is USD 400/month. The cost of erythropoietin is similar to that of MHD. The cost of RT varies between the government-run and private sector. The cost of the RT procedure is USD $800-1,000$ in government-run hospitals and USD 6,000-7,000 in the private sector. The cost of immunosuppression using triple immunosuppression (tacrolimus, steroid and mycophenolate) is USD $350-400 /$ month, and may decrease if the calcineurin inhibitor is withdrawn after the initial few months and the patient is only on steroid and mycophenolate or azathioprine. Thus, on a long-term basis, RT still results in being the cheaper modality of treatment for ESRD in India when compared to dialysis. With the availability of automated cyclers for automated peritoneal dialysis, the availability of newer immunosuppressive medications like rapamycin, IL-2 receptor antibodies, etc., the cost of therapy has also increased. Some of the induction immunosuppression, like IL-2 receptor antibody use, is more on the basis of criteria other than the medical. In the absence of state-funded medical treatment and medical insurance facilities being minimal for CKD/ESRD patients, there are various sources where patients get money for treatment, e.g. help from an employer/charity, loan, selling assets, or pooling their family resources. Obviously, many have more than one source. It is not difficult at all to imagine what, if only $10 \%$ of ESRD patients get therapy, is going to happen to the rest of them.

Thus, planning for prevention of CKD on a long-term basis is the only practical solution for India, as probably also for any other country. CKD is accepted to be an illness for which a prevention program should be applied, as it is highly prevalent in the society, it can be detected easily, and the cost of prevention is much less than the treatment.

\section{Progress and the Current Status in the Management and Prevention of CKD}

\section{Progress in Management of CKD}

$\mathrm{CKD}$ is considered like any other chronic disease when it comes down to developing strategies for prevention and management. Obviously, it is bracketed under one group of chronic diseases for any health planner. Until recently, the Indian public health system did not recognize CKD as being a significant problem. Other non-communicable diseases like cancer, CVD, and accidents were receiving the main focus of public health plans and programs. Therefore, there was no dedicated funding for prevention and management of CKD by the Health Ministry of the Government of India. The media have often reported about the situation, particularly the infamous kidney racket episode drew the attention of the government towards CKD, and it was felt that CKD and ESRD require focused attention. Academic activities were started in 2005 on different aspects of CKD. Important meetings were organized by AIIMS in collaboration with ICMR, Planning Commission and International Society of $\mathrm{Ne}$ phrology in 2005 and 2008. These meetings made specific recommendations for CKD prevention. Further, the government has also realized the increasing financial burden on account of dialysis and other related treatments for its Central Government Health Services beneficiaries and decided to go for an experimental program of hemodialysis on internationally approved protocolbased stand-alone dialysis unit patterns. All these actions have created a general awareness in the public health system, particularly at the policymaker and planner level, that CKD requires focused attention. However, seeing the magnitude of CKD, more actions are required at governmental level for all the CKD problems in India. An account of important development in CKD is given below.

The government has initiated a process in which it is planning to establish stand-alone hemodialysis units. These units are being discussed with the aim of establishing in public-private partnership an increase of facilities at an affordable cost. Consultations with nephrologists have taken place, and they started the exercise by developing a 12-month training program for dialysis physicians, so as to produce trained physicians to man the dialysis units. The shortage of nephrologists necessitated this exercise because a nephrologist is essential for a dialysis unit. Negotiations are ongoing to find suitable partners for stand-alone dialysis units. This exercise is aiming at the prevention and maintenance of the patient's condition, so that they can undergo renal transplantation when a suitable organ/donor becomes available. However, this activity is at a very preliminary stage and will still require a lot more time before it will be in place for patient use.

On the RT side, the government has launched a National Organ Transplant Program (NOTP) to facilitate both living related and cadaver organ transplantation. A dedicated budget has been provided for it. NOTP will take care of diseased organ donation, centralized organ procurement and the distribution system, and capacity building for organizing renal transplantation units on a national scale. Coupled with amendments of the Transplantation of Human Organ Act (THOA), this action of the Ministry of Health and Family Welfare (MOHFW) 
will reduce the gap between demand and supply of organs, especially kidneys, thus providing final treatment for CKD patients.

A large number of patients, medical personnel and the public at large were of the opinion that the current THOA has many lacunae and there is need for change in the act and its rules. Many meetings between medical personnel, judiciary, NGO and policymakers as well as a national consultation have taken place in recent years to decide on the changes in the act and rules within it. At many of these meetings, recommendations had been put on the Ministry of Health's website for the public to comment. A final draft for changes was made and put on the Ministry's website (http://mohfw.nic.in/THOA\%20-\%20Cab inet $\% 20$ Note $\% 20$ Modified.pdf) for the public at large to see and comment. The few major proposed changes, which are being interpreted to increase the RT, include grandparents in the definition of 'near relatives', making it mandatory for the ICU/treating medical staff to request relatives of brain-dead patients for organ donation, inclusion of an anesthetist/intensivist for declaration of brain death in the event of the non-availability of a neurosurgeon/neurologist, allowing swap operations for RT on a case-to-case basis, creating a position of transplant coordinator in every hospital recognized for doing RT, some incentives (not payment) to living related donors and to relatives of cadaver donors, and establishment of national registry related to organ transplant. However, it will take some time to bring about a visible change in the situation of CKD treatment.

\section{Progress in the Prevention of CKD}

Prevention of any illness needs patience. It should also be a priority area for any government. From that perspective, CKD prevention is still far away. There are certain facts which need to be kept in mind. Public health expenditure in India is around $1 \%$ of the gross domestic product (GDP), with a central share of $30 \%$ and a state share of $70 \%$. As India's GDP is constantly increasing, even if public health expenditure is being maintained at $1 \%$ of the GDP, the actual public sector expenditure shows a gradually increasing trend every year. However, this increase is very low in comparison to the needs and requirements of the health sector. Therefore, with limited budgetary support, we are organizing a prevention program. Vertical health and family welfare programs are getting adequate synergization at operational levels through the National Rural Health Mission (NRHM). A focused Information, Education and Communication (IEC) campaign for $\mathrm{CKD}$ is being planned through various related national programs, i.e. National Programme of Diabetes Mellitus, CVD, stroke and NOTP. All secondary prevention activities are being taken care by the National Programme of Diabetes Mellitus, CVD, Stroke \& NRHM. The government has initiated NRHM (2005-2012) to provide effective healthcare to rural populations throughout the nation with special focus on the states, which have weak public health indicators, and with the aim of raising public spending on health from 0.9 to $2-3 \%$ of the GDP. It does include prevention and control of non-communicable diseases. However, CKD is still not in the focus. It can be borne by the fact that last year (2008) the government launched a pilot project of the National Program for Prevention and Control of Diabetes, Cardiovascular Diseases and Stroke (NPCDS) in seven of the nation's states. The total plan allocation envisaged for an ongoing 5-year plan is likely to be USD 3.8 million. Being a common risk factor, CKD could only find an indirect focus. The website of 'Healthy India' (http://healthy-india.org/) does not have prevention of CKD as a dominant disease on its homepage, while diabetes, heart disease, cancer, hypertension and strokes are present. Thus, India has a long way to go before CKD and ERSD get their rightful place in preventive programs of the Government of India.

In a recent study conducted by AIIMS in the first-degree relatives of non-diabetic CKD patients has shown a very high incidence of CKD and its risk factors [unpubl. data]. The prevalence of CKD was 9.94\%, low eGFR 5.43\% and proteinuria $6.47 \%$. The prevalence of hypertension, diabetes, impaired fasting glucose, and hyperuricemia was $31.37,4.19,22.36$ and $14.4 \%$, respectively. Among those who were detected to have kidney disease or its risk factors in this study, the awareness of their condition was very low even though they had a family member with ESRD. Thus, in a country like India, where screening and prevention of CKD still has to take a start, screening of high-risk individuals for CKD and its risk factors is the best bet.

To conclude, CKD is a problem of epidemic proportions in India, and with an increasing diabetes burden, hypertension, and growing elderly population it is going to increase even further. Managing the patient population of CKD even with better organization of RRT will be impossible. The money invested at this time in establishing a prevention program for CKD is definitely going to give results in years to come and ultimately in the long run will still be cost-effective. This money can be utilized for other healthcare programs. However, it requires a lot of data and professional lobbying with various policymakers, the MOHFW, and the Government of India. 


\title{
References
}

1 World Health Organization: Preventing Chronic Disease: A Vital Investment. Geneva, WHO, 2005.

$\checkmark 2$ Grassmann A, Gioberge S, Moeller S, et al: ESRD patients in 2004: global overview of patient numbers, treatment modalities and associated trends. Nephrol Dial Transplant 2005;20:2587-2593.

3 Center for Disease Control and Prevention (CDC): Prevalence of chronic kidney disease and associated risk factors - United States, 1999-2004. MMWR Morb Mortal Wkly Rep 2004;56:161-165.

4 Hamer RA, El Nahas AM: The burden of chronic kidney disease. BMJ 2006;332:563564.
5 Agarwal SK, Dash SC, Irshad M, et al: Prevalence of chronic renal failure in adults in Delhi, India. Nephrol Dial Transplant 2005;20: 1638-1642.

6 Mani MK: Prevention of chronic renal failure at the community level. Kidney Int 2003; 63(suppl 83):S86-S89.

7 Mani MK: Experience with a program for prevention of chronic renal failure in India. Kidney Int 2005;67(suppl 94):S75-S78.

$\checkmark 8$ Modi GK, Jha V: The incidence of end-stage renal disease in India: a population-based study. Kidney Int 2006;70:2131-2133.

-9 Mani MK: Nephrologist sans frontières: preventing chronic kidney disease on a shoestring. Kidney Int 2006;70:821-823.
10 Agarwal SK, Dash SC: Spectrum of renal diseases in India in adults. J Assoc Physicians India 2000;48:594-600.

$>11$ Mani MK: Chronic renal failure in India. Nephrol Dial Transplant 1993;8:684-689.

$>12$ Mittal S, Kher V, Gulati S, Agarwal LK, Arora P: Chronic renal failure in India. Ren Fail 1997; 19:753-770.

13 Sakhuja V, Jha V, Ghosh AK, Ahmed S, Saha TK: Chronic renal failure in India. Nephrol Dial Transplant 1994;9:871-872.

14 Dash SC, Agarwal SK: Incidence of chronic kidney disease in India. Nephrol Dial Transplant 2006;21:232-233.

15 Agarwal SK: Chronic kidney disease and its prevention in India. Kidney Int 2005;98: S41-S45.

\section{Editorial Comment}

\author{
M. El Nahas, Sheffield
}

The minireview by Agarwal and Srivastava highlights very clearly the issues facing patients with CKD in India and reflects more generally those facing patients with ESRD in emerging economies. An increase in the number of patients with diabetes and hypertension combined with poverty has led to an upsurge of patients with CKD. On the other hand, according to the authors, only $10 \%$ of patients with ESRD have access to RRT. Most dialysis units are privately run and the cost of dialysis beyond the means of most Indians. Transplantation in India has been fraught by issues including organ trafficking. Such a discrepancy between demand and supply highlights the need for concerted policies. Individual initiatives have started to address screening those at risk of CKD including diabetics and hypertensive as done in Chennai and elsewhere. But more significantly, the Indian government has taken two major initiatives to address CKD and ESRD, namely providing government-sponsored dialysis facilities as well as a national organ transplantation program. The Indian government ought to be congratulated for these strategic steps towards improving the healthcare of CKD patients. It provides a template for many emerging nations and governments to follow. The Indian experience in the field of ESRD and transplantation will be closely followed by all those who care for CKD in the developing world. 